Esau, K. 1965a. Vascular differentiation in plants. Holt, Rinehart and Winston, New York. - 1965b. Plant anatomy. 2nd ed. John Wiley and Sons, Inc., New York.

Ezelarai, G. E., and K. J. Dormer. 1963. The organization of the primary vascular system in the Ranunculaceae. Amn. Bot. 27: 23-38.

Fahn, A., ANd I. W. Baitey. 1957. The nodal anatomy and the primary vascular cylinder of the Calycanthaceae. J. Arnold Arbor. 38: 107-117.

Jacons, W. P., AND I. B. Morrow. 1957. A quantitative study of xylem development in the vegetative shoot apex of Coleus. Amer. J. Bot. 44: 823-842.

JEFFreY, E. C. 1917. The anatomy of woody plants. University of Chicago Press.

KumarI, G. K. 1963 . The primary vascular system of gymnosperms. Ph.D. thesis. University of Michigan, Ann Arbor.

Money, L. L., I. W. BaIley, and B. G. Swamy. 1950. The morphology and relationships of the Monimiaceae. J. Arnold Arbor. 31: 372-404.

Philipson, W. R., And E. E. Balfour. 1963. Vascular patterns in dicotyledons. III. Anatomy of the stem of Calycanthaceae. Trans. Roy. Soc. Edinb. 52: $517-530$.

QUinian, C. E. 1920. Contributions towards a knowledge of the anatomy of the lower dicotyledons. III. Anatomy of the stem of Calycanthaceae. Trans. Roy. Soc. Edinb. 52: 517-530.

Wetmore, R. H., and C. W. Wardlaw. 1951. Experimental morphogenesis in vascular plants. Ann. Rev. Plant Physiol. 2: 269-292.

Amer. J. Bot. 54(7): 813-820. 1967.

\title{
DEVELOPMENTAL PATTERNS IN STEM PRIMARY XYLEM OF WOODY RANALES. II. SPECIES WITH TRILACUNAR AND MULTILACUNAR NODES ${ }^{1}$
}

\author{
David H. Benzing \\ Department of Botany, University of Michigan, Ann Arbor²
}

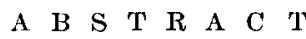

\begin{abstract}
Patterns of early protoxylem strands in vegetative stems of 16 species of woody Ranales representing nine families with trilacunar or multilacunar nodes are illustrated and discussed. The order of differentiation of first protoxylem in all examined species is basipetal. Open, protoxylem strand patterns are more common than closed patterns in these taxa and 11 other woody ranalean species with unilacunar nodes. Protoxylem strand patterns in woody Ranales are diverse, even among species with identical phyllotaxis and nodal anatomy. Pattern similarities of possible taxonomic significance exist, however, between some species of the Annonaceae and Myristicaceae, Hernandiaceae and Lauraceae, and the Canellaceae and Winteraceae. The general diversity of strand patterns in woody Ranales may reflect the high degree of evolutionary isolation among ranalean families. Results of these investigations on stem anatomy of woody Ranales indicate that the two-trace, unilacunar node is probably not primitive in the Angiospermae. Evidence from this study and others suggests that the primitive node in the Angiospermae, and possibly in all seed plants, is one-trace, unilacunar or trilacunar. Two-trace, unilacunar nodal anatomy of the Clerodendron type may have evolved in conjunction with the evolution of decussate phyllotaxis. The protoxylem strand patterns of Illicium and/or Drimys are probably the most primitive of those described in the woody Ranales.
\end{abstract}

IN A PRECEDING STUDY (Benzing, 1967) patterns of protoxylem strands in vegetative shoots of 11 woody ranalean species characterized by unilacunar nodal anatomy were described. These

${ }^{1}$ Received for publication 1 June 1966.

This work was based on a dissertation submitted to the Graduate School of The University of Michigan in partial fulfillment of the requirements for the degree Doctor of Philosophy.

Sincere appreciation is extended to Dr. Charles B. Beck and Dr. Warren H. Wagner, Jr., for their advice during the course of this research and in the preparation of this manuscript. Appreciation is also extended to the collectors mentioned in Table 1.

2 Current address: Department of Biology, Oberlin College, Oberlin, Ohio. patterns are diverse and often differ among species possessing identical phyllotaxis. In this paper patterns of protoxylem strands of 16 woody ranalean species showing trilacunar and multilacunar nodes are considered. Materials, methods, and terminology utilized here have been discussed in the previous paper.

Observations-Annona muricata, Asimina triloba, Cananga odorata (Annonaceae), $M y$ ristica fragrans (Myristicaceae)-The phyllotaxis and protoxylem strand pattern of vegetative shoots of the three members of the Annonaceae and the single member of the Myristicaceae 

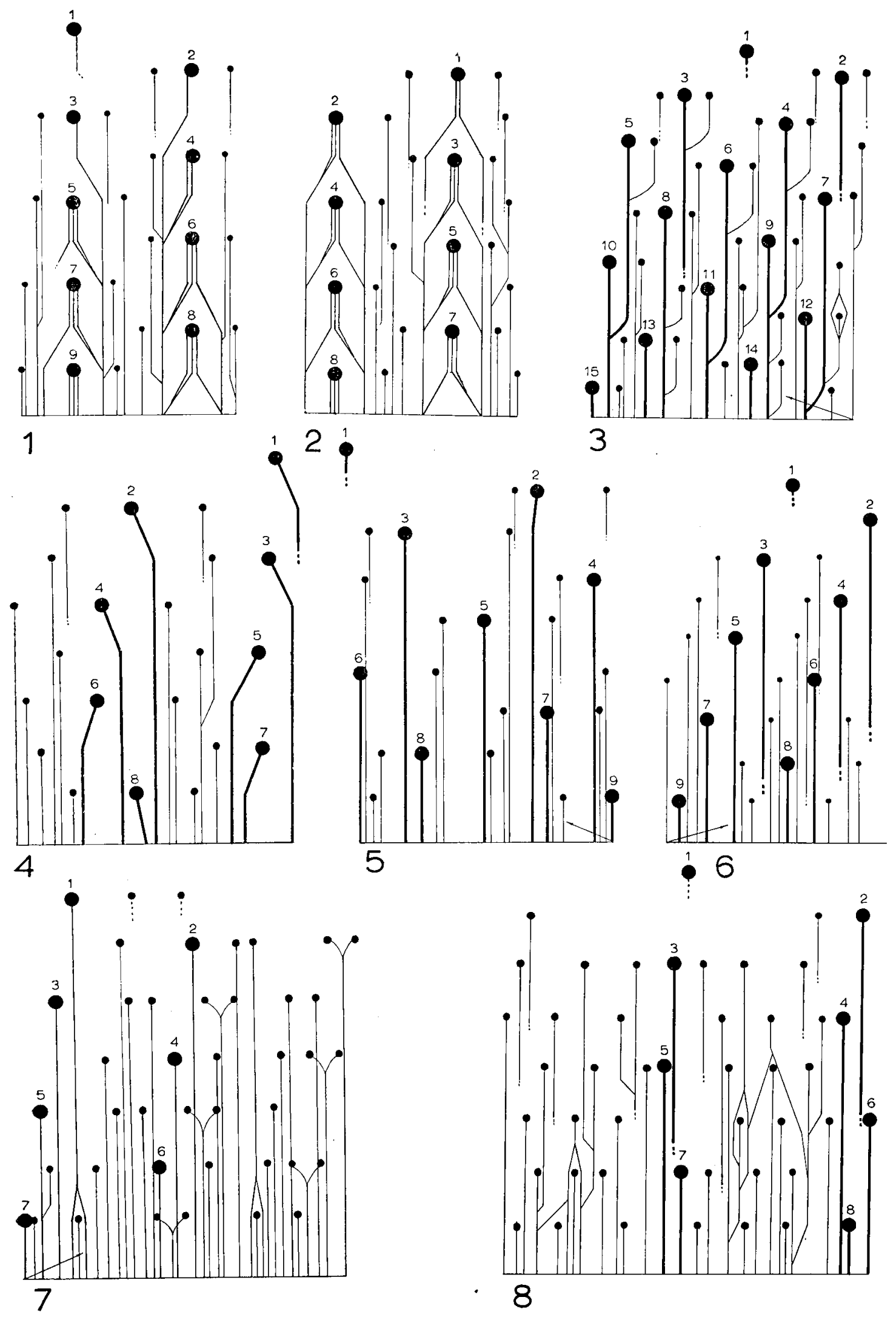
investigated in this survey are sharply distinct from those of the ranalean species discussed in the previous paper (Benzing, 1967). Leaf arrangement exhibited by these four species is distichous, and the nodal anatomy is trilacunar (Table 1 ). The strand patterns representing Annona muricata and Asimina triloba (Fig. 2) and Cananga odorata (Fig. 1) reflect the relationship between strand pattern and phyllotaxis.

In vegetative stems of all four species, two pairs of scorpioid sympodia exist; each pair produces median leaf-trace strands to a particular rank of leaves. The strand contributed by one scorpioid sympodium of a pair in Annona muricata, A simina triloba and Cananga odorata divides into two; the contribution from the other sympodium of the pair remains single. Hence, at departure from the vascular cylinder, the initial protoxylem vascularization to the young leaf from the central gap consists of three closely placed strands. In contrast, Myristica fragrans exhibits a single median leaf-trace strand which results from the fusion of two unbranched strands from a sympodial pair. This fusion product then passes out through the central gap as the median trace strand. Cananga odorata (Fig. 1), unlike Annona muricata, Asimina triloba and Myristica fragrans, exhibits a consistent delay in the development of one of each pair of sympodia furnishing median leaf-trace strands.

The lateral leaf-trace strands in all four species form two groups positioned between the two pairs of scorpioid sympodia. One lateral leaf-trace strand is furnished to every leaf by each of the two lateral strand groups. In a few instances lateral strands arise from the scorpioid sympodia. More originate from pre-existing lateral strands, and the pattern is basically open. At a given level, median leaf-trace strands show earlier basipetal differentiation of first protoxylem elements than the lateral leaf-trace strands.

Bubbia sp., Drimys colorata, Drimys winteri, (Winteraceae), Canella alba, Warburgia ugandensis (Canellaceae) - The configuration of the protoxylem strand pattern of Drimys winteri (Fig. 3) is also characteristic of Drimys colorata, Canella alba, Warburgia ugandensis and, to a lesser extent, Bubbia sp. Therefore, a discussion of the strand pattern of Drimys winteri and additional comments concerning Bubbia sp. will suffice for all five species.

Five distinct helicoid sympodia are apparent in Drimys winteri stems. Each sympodium gives rise to both median and lateral leaf-trace strands. Leaf-trace strand divergence is exclusively cathodic whether the ontogenetic spiral is sinistrorse or dextrorse. The number of helicoid sympodia agrees with the denominator of the $2 / 5$ phyllotactic fraction observed for all five of these species. Three-trace, trilacunar nodal anatomy is common to all five species. Additional comments on the stem morphology of the Canellaceae and the Winteraceae have been published by Wilson (1960) and Bailey and Nast (1945), respectively.

Figure 3 reveals the consistently repeating nature of this strand pattern. Every fifth leaf on the axis is supplied by a median leaf-trace strand from the same sympodial system. Lateral leaftrace strand vascularization of each leaf is also regular. Every leaf is supplied by one lateral strand from the same sympodium producing the median strand to that leaf. The other lateral strand arises from the sympodium on the anodic side of the sympodium furnishing the median trace strand to that leaf (see node 12, Fig. 3). The length of median and lateral leaf-trace strands is irregular. Occasional lateral strands fuse with other laterals and the common strand then fuses with a sympodium at a lower level. Of the five species of the Winteraceae and Canellaceae investigated, only Bubbia sp. exhibits anastomoses between adjacent sympodia. The resulting increase in strand number per stem cross-section may be related to the unusually large stem diameter characteristic of young twigs of this plant.

Himantandra belgraveana (Himantandraceae)Two types of leaf arrangement with correspondingly distinct patterns of protoxylem strands were found in vegetative shoots of $H$. belgraveana. The strand pattern of shoots possessing distichous phyllotaxis is illustrated in Fig. 4. The strand pattern of other shoots from the same collection exhibiting $2 / 5$ spiral leaf arrangement is indicated in Fig. 5. Distichous shoots of $H$. belgraveana possess a grouping of median and lateral leaftrace strands. Shoots with spiral phyllotaxis show an interspersion of median and lateral leaf-trace strands. Both patterns are open and lack sympodia, although occasional fusions occur between lateral leaf-trace strands. The threetrace, trilacunar nodal anatomy of this species has been described bv Bailey and Smith (1942).

Fig. 1-8. Solid lines depict protoxylem strands made up of mature xylem elements. Dotted lines represent strands devoid of fully differentiated xylem elements. Lines terminated by large black circles are median leaf trace xylem strands. Lines terminated by smaller black circles are lateral leaf trace xylem strands. The arrows indicate the direction of the ontogenetic spiral.-Fig. 1. Cananga odorata.-Fig. 2. Asimina triloba and Annona muricata.-Fig. 3. Drimys minteri.Fig. 4. Himantandra belgraveana shoot with distichous phyllotaxis.-Fig. 5. Himantandra belgraveana shoot with spiral phyllotaxis.-Fig. 6. Hibbertia scandens.-Fig. 7. Michelia figo shoot with dorsiventral phyllotaxis.-Fig. 8. Eupomatia laurina. 
Hibbertia scandens (Dilleniaceae)-The protoxylem strand pattern of Hibbertia scandens (Fig. 6) is an open one in which the three strands entering each leaf exit from the vascular cylinder through three widely separated gaps. The alternate leaves in ortho-distichous arrangement form two parallel parastichies or spirals along the axis (in distichous phyllotaxis the leaves are alternate in placement and two ranked). These parastichies can be visualized if each strand termination in the two groups of median leaftrace strands winding around the axis is considered a point of leaf attachment. The angle of divergence between successive leaves on the same spiral is approximately $50 \mathrm{deg}$ (Table 1). Alternating with the two spirals of median leaftrace strands are two spirals of lateral leaf-trace strands. A single group of median strands provides the median leaf-trace strand to every other leaf on the axis or every leaf in one of the two parastichies, while one lateral strand is furnished to every leaf from each of the two lateral strand groups. Strands making up the four strand groups do not fuse to form sympodia and the pattern is open.

Michelia figo (Magnoliaceae)-Shoots of $M i$ chelia figo available to the author displayed dorsiventral phyllotaxis, although some shoots with spiral phyllotaxis have been reported in this species by Tucker (1962). Figure 7 illustrates the early protoxylem strand pattern of a vegetative shoot with dorsiventral leaf arrangement. As in Himantandra belgraveana, only occasional fusions occur among the strands and the pattern is basically open.

The number of lateral leaf-trace strands vascularizing each leaf and the associated stipule varies in Michelia figo. Similar inequalities in nodal structure have been reported for other members of the Magnoliaceae by Canright (1955) and Ozenda (1949). Five lateral leaf-trace strands normally depart from the vascular cylinder at most nodes of $M$. figo, but one is occasionally missing. In a single instance seven strands were observed vascularizing a leaf. In the examined material the lateral leaf-trace strand departing from the vascular cylinder at a point opposite the departure of the median leaf-trace strand divides into two just after entering the cortex. These two strands then enter the sheathing stipule. All other lateral strands encircle the vascular cylinder through the cortex and enter the leaf base. First appearance of mature tracheary elements below the shoot apex occurs in the median leaf-trace strand. Mature protoxylem elements then appear in the lateral leaf-trace strands closest to the median strand and lastly in the lateral strands farthest from the median strand.

Eupomatia laurina (Eupomatiaceae)-All shoots of Eupomatia laurina obtained by the author exhibited distichous leaf arrangement. As Fig. 8 indicates, protoxylem strand vascularization of leaves in $E$. laurina consists of seven strands arising from seven equally separated gaps encircling the vascular cylinder. Occasionally lateral leaf-trace strands farthest from the median leaf-trace strand are weakly developed or fail to develop at all. Examination of the paths of the leaf-trace strands reveals a lack of any well-developed sympodial branching. Occasional fusions occur between lateral leaf-trace strands, but they are not a regular feature of the pattern. Differentiation of first xylem elements in the strands at a particular level follows that described above for Michelia figo.

Magnolia virginiana (Magnoliaceae)-All shoots investigated by this author possessed $2 / 5$ phyllotaxis, although other leaf arrangements have been reported for the genus. The nodal anatomy of $M$. virginiana is very similar to that of Michelia figo with six to eight lateral leaftrace strands in addition to the median leaftrace strand departing from the vascular cylinder at each node. In contrast to Michelia figo, leaftrace strands of Magnolia virginiana do not depart from gaps completely encircling the vascular cylinder. At some nodes a strand branches off a lateral leaf-trace strand as the latter passes out through the cortex (Fig. 9). This branch then remains in the internodal cortex to the level of the next younger node. Here the single strand branches and all the derivative strands enter the stipule.

Interconnection of strands in the vascular cylinder is uncommon and irregular, and welldefined sympodia are absent. Median leaf-trace strand divergence, when present, is always anodic, while lateral leaf-trace strand divergence may be either anodic or cathodic though predominantly anodic. The strand pattern is open since most strands fail to connect with other strands.

Degeneria vitiensis (Degeneriaceae)-The phyllotaxis of Degeneria vitiensis (Fig. 10) is similar to that described earlier for Hibbertia scandens. Protoxylem vascularization of each foliar appendage on stems of Degeneria vitiensis consists of five strands arising from five widely separated gaps around the vascular cylinder. The origin of lateral leaf-trace strands is regular, some fusing with median strands. Certain lateral strands give rise in the anodic direction to other lateral strands supplying the succeeding leaf above. Median strands are not connected to each other. First appearance of xylem elements in the developing leaf-traces follows the sequence described above for other multilacunar species. The nodal anatomy of $D$. vitiensis has been described by 'Swamy (1949). 


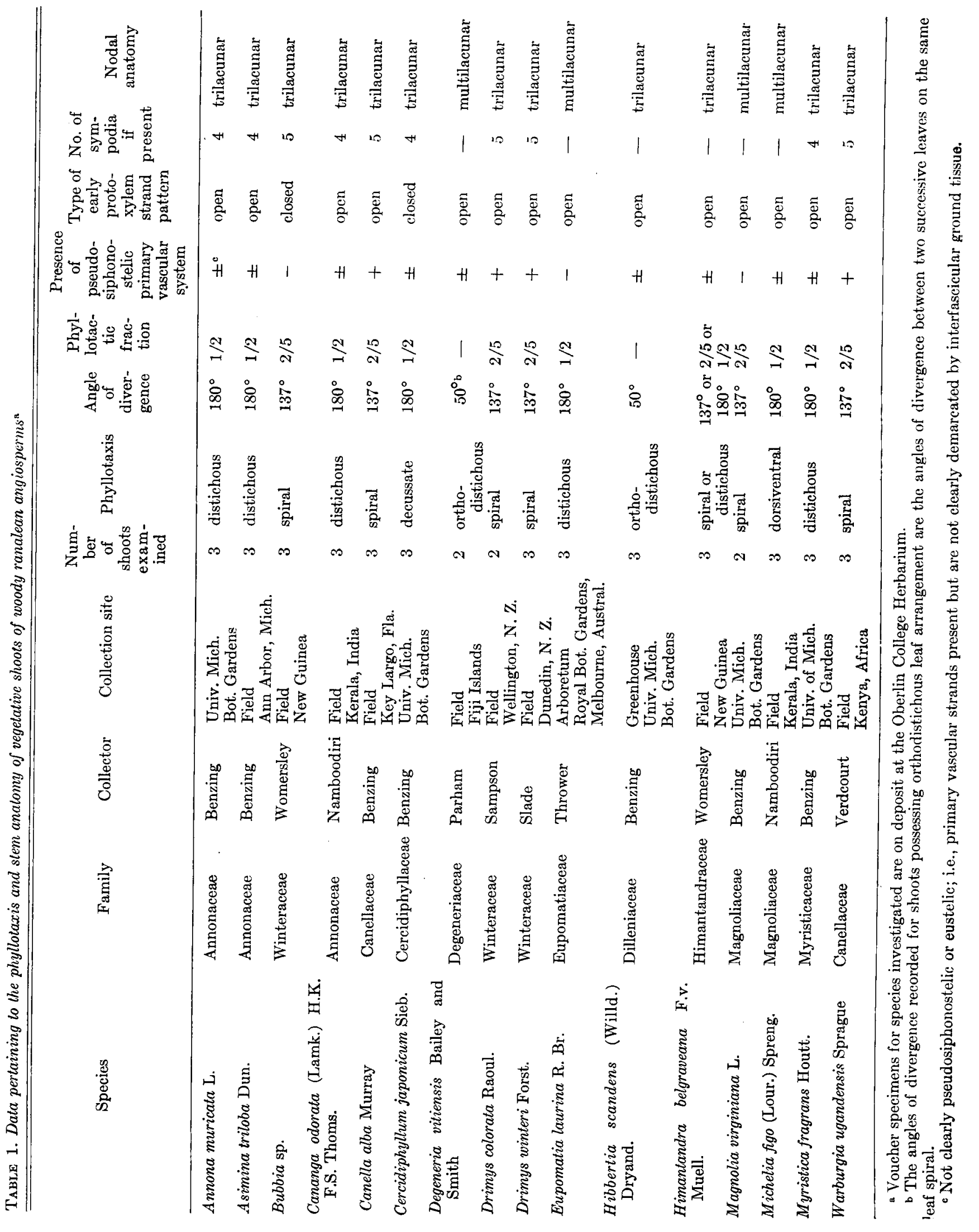




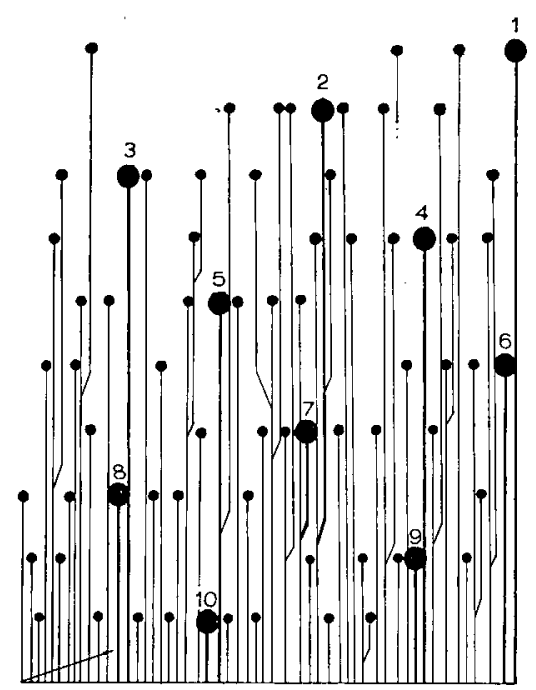

9

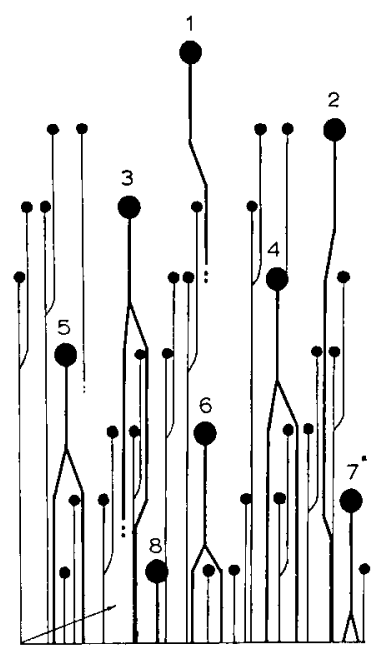

10

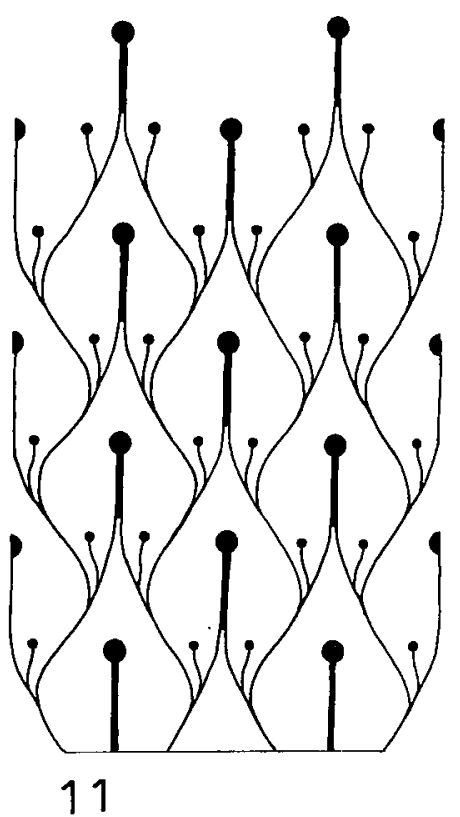

11

Fig. 9-11.-Fig. 9. Magnolia virginiana.-Fig. 10. Degeneria viliensis.-Fig. 11. Cercidiphyllum raponicum.

Cercidiphyllum japonicum (Cercidiphyllaceae)The closed protoxylem strand pattern of Cercidiphyllum japonicum is illustrated in Fig. 11. Vegetative shoots of this species exhibit decussate leaf arrangement and a very regular strand pattern. Nodes of mature stems of C. japonicum have been described as three-trace, trilacunar (Bailey and Swamy, 1949).

Four scorpioid sympodia make up the basic structure of the strand pattern of $C$. japonicum. Each of these scorpioid sympodia produces a part of the single median leaf-trace strand supplying each leaf. The direction of divergence of these contributions to the median leaf-trace strand alternates at each node. Since the two contributions to the median strand fuse, thus joining adjacent sympodia, the pattern is closed. The regular sinuate pathway of these sympodia is correlated with median strand production. Lateral strands to a particular leaf originate from the basal region of the two strands that will fuse to comprise the median strand one node above. Median strands traverse nearly two nodes before entering a leaf, whereas lateral strands are much shorter.

Discussion-A majority of woody ranalean species investigated by the author (see also Benzing, 1967) exhibit a continuous or nearly continuous ring of procambium, primary phloem and, later, of primary xylem close behind the shoot apex. Such a primary vascular system is termed pseudosiphonostelic (Bailey and Nast, 1948). Unlike the primary phloem, the protoxylem tissue is tangentially discontinuous for a considerable distance down the growing shoot axis of pseudosiphonostelic stems. The longitudinal direction of development of the initially discrete protoxylem strands is basipetal. In three-trace and multitrace systems, differentiation of the earliest tracheary elements always occurs in the median leaf trace strand and later in lateral leaf trace strands. The tangentially discontinuous strands of protoxylem tissue exhibit species specific patterns which can be reconstructed by careful analysis of serial sections of growing shoot tips. These patterns are highly diverse among the 27 species investigated.

More morphological similarity in the protoxylem strand pattern among different species of the Ranales was expected, since many characters of vascular tissue anatomy have been shown to be conservative enough to be of great taxonomic value above the species level. The general uniformity in primary vascular bundle pattern throughout a fairly diverse group of plants such as the case reported in the Coniferales by Iiumari (1963) has no parallel in the early protoxylem strand patterns of the investigated woody Ranales. The basis of the diversity among protoxylem strand patterns in woody ranalean flowering plants is unclear, but a few possibilities can be ruled out.

Factors which do not regularly affect the strand pattern include phyllotaxis, leaf size and form, and, to a lesser extent, nodal anatomy. Various pattern types do not necessarily correspond to the several types of phyllotaxis found in the ranalean complex. To illustrate, the shoots of Illicium parviflorum, Persea americana, Mag- 
nolia virginiana, Schisandra glabra and Drimys winteri studied by the author are characterized by the common $2 / 5$ phyllotaxis', approximately the same angle of divergence, and simple leaves with pinnate venation-yet each exhibits a different strand pattern. Consequently, the position of leaf primordia at the shoot tip alone does not govern all developmental aspects of the subjacent early protoxylem tissue. Likewise, strand pattern diversity from taxon to taxon does not seem to correlate with leaf size, since all the above-mentioned species possess leaves of moderate proportions.

Further, a particular type of nodal anatomy is not always associated with a specific protoxylem strand pattern. This study and the related one (Benzing, 1967) reveal that much diversity in strand pattern may exist among species characterized by a single type of nodal anatomy. For example, Hibbertia scandens, Cercidiphyllum japonicum, Myristica fragrans, Drimys colorata, and Himantandra belgraveana are characterized by three-trace trilacunar nodes (at maturity), whereas an analysis of the protoxylem reveals a strong pattern diversity in this tissue system. The greater morphological consistency of the primary vascular system at the mature node may be related to the uniform structure of most ranalean leaves and leaf bases.

Variability in the protoxylem strand pattern among woody Ranales is no doubt in part a reflection of the extreme evolutionary divergence of the families within the order. The great diversity and unique combinations of vegetative and floral characteristics tend to substantiate this idea. The often proposed hypothesis that the Ranales represent a group of terminal relicts of a once much larger and ancient assemblage of plants which may have occupied a dominant position in a past angiosperm or proangiosperm flora before the emergence of more modern flowering plant types seems plausible. Although the above theory is appealing, the final resolution of the problem must await further understanding of the nature and evolution of the genetic and morphogenetic mechanisms controlling the development of tissue systems such as the protoxylem.

The closed protoxylem strand pattern tends to correlate with advanced or specialized vegetative and floral characteristics. Open, protoxylem strand patterns are found in the Degeneriaceae, Illicaceae, Magnoliaceae, Schisandraceae and Winteraceae-families considered among the most primitive of the Ranales by virtue of many vegetative and floral characters. In contrast, Calycanthus, Cercidiphyllum, and Dillenia have closed strand patterns and many floral and vegetative characters considered to be advanced. These latter taxa possess decussate or whorled leaf arrangement, uncommon phyllotaxes in the Ranales, which are probably derived from the more widespread spiral leaf arrangement. This situation seems to parallel that in the conifers where only species characterized by opposite leaf arrangement possess closed primary vascular bundle systems (Kumari, 1963). Here, however, the comparison of vascular patterns is not really valid since conifers are eustelic. A majority of the woody ranalean species investigated possess open protoxylem strand patterns.

Among species investigated in a single family, protoxylem strand patterns are generally consistent. However, since many ranalean families are small, or, if large, are represented in this survey by only a few species, such an observation is tenuous. In at least one of the larger families (Dilleniaceae), considerable divergence in strand pattern is exhibited between Dillenia (Fig. 20, Benzing, 1967) and Hibbertia (Fig. 6), but this may be correlated with the widely diverse patterns of phyllotaxis in the Dilleniaceae.

From this survey of a few species and the findings of other investigators, it is difficult to decide which strand pattern, if any, approximates the ancestral type. Likewise, trends and derived types are impossible to identify with any degree of certainty. The structurally simple protoxylem strand pattern of Illicium parviforum may be most primitive. Bailey and Nast (1948) point out that Illicium is strongly pseudosiphonostelic - the continuous vascular cylinder being broken only by a single leaf gap at each node. Because it is strongly pseudosiphonostelic, the primary vascular system of Illicium is unlike that of most higher angiosperms.

A protoxylem strand pattern such as that of Drimys winteri might be basic and the pattern of Illicium parviflorum derived by the loss of the lateral leaf-trace strands and the associated gaps. A parallel proposal was made by Sinnott (1914) concerning the evolution of nodal anatomy.

Determination of the ancestral type of nodal structure in the primitive angiosperms is equally difficult since both the trilacunar and unilacunar types are common among these plants. The multilacunar type is restricted to only three ranalean families and probably represents a specialized type. The occurrence of plants with the two-trace, unilacunar nodal structure proposed as primitive by Marsden and Bailey (1955) and Bailey (1956) is limited to a few ranalean families characterized by derived decussate phyllotaxy and many specialized floral characters (Chloranthaceae, Monimiaceae sensu lato, Austrobaileyaceae). The possibility that two-trace, unilacunar nodal anatomy evolved in association with opposite phyllotaxy from an ancestral condition characterized by alternate leaf arrangement and possibly an open protoxylem strand or primary vascular bundle pattern is quite likely. The presence of few gymnosperms and progymnosperms in the fossil record with decussate phyllotaxis and/or Clerodendron type two-trace, uni- 
lacunar nodal anatomy indicates that this type of nodal structure is not basic in the seed plants (Kumari, 1963; Pant and Mehra, 1964). The anatomy of cotyledonary nodes does not necessarily reflect ancestral conditions in the mature stem; the unique seedling morphology and decussate insertion of the cotyledons make this unlikely. Taking all available information into consideration, the odd-number trace, unilacunar and/or the three-trace, trilacunar nodes are more likely to be primitive in the Angiospermae than the Clerodendron node.

Although the protoxylem strand patterns of vegetative stems among woody ranalean angiosperms investigated are diverse, a few significant similarities exist. Some of the similarities between strand patterns of different species probably result from parallelism or convergence, while others may indicate common origin. The distinction between these two is by no means easy to make. The nearly identical strand patterns among the few species of the Annonaceae and Myristicaceae studied by the author may denote affinities between the two families, but the sample is very small. Similarities in pollen grain development (Joshi, 1946), wood anatomy (Vander Wyk and Canright, 1956), and the presence of ruminate endosperm in both the Annonaceae and Myristicaceae favor this possibility. Ruminate endosperm, however, has also been reported in the Canellaceae (Parameswaran, 1961). Similarity among strand patterns in the species of the Canellaceae and Winteraceae investigated indicates possible relationship between these two families. Similarities in early protoxylem strand patterns among investigated species of Hernandiaceae, Eupteleaceae, and Schisandraceae and among the Lauraceae, Eupteleaceae, and Schisandraceae may represent parallelism, since consideration of characters from all organ systems indicates but little relationship among these taxa. Contrariwise, strand pattern similarities between Hernandia and Lindera favor proposed relationships between the Hernandiaceae and Lauraceae (Stern, 1954; Shutts, 1960).

\section{LITERATURE CITED}

Bailey, I. W. 1956. Nodal anatomy in retrospect. J. Arnold Arbor. 37: 269-287.
morphology of the Winteraceae. VII. Summary and conclusions. J. Arnold Arbor. 26: 37-47.

- , AND $\longrightarrow$. 1948. Morphology and relation ships of Illicium, Schisandra, and Kadsura. I. Stem and leaf. J. Arnold Arbor. 29: 77-89.

$\longrightarrow$ - AND A. C. Smith. 1942. Degeneriaceae, a new family of flowering plants from Fiji. J. Arnold Arbor. 23: 356-365.

—, And B. G. L. Swamy. 1949. The morphology and relationships of Cercidiphyllum. J. Arnold Arbor. 30: $187-210$.

Benzing, D. H. 1967. Developmental patterns in stem primary xylem of woody Ranales. I. Species with unilacunar nodes. Amer. J. Bot. 54: 805-813.

Canright, J. E. 1955. The comparative morphology and relationships of the Magnoliaceae. IV. Wood and nodal anatomy. J. Arnold Arbor. 36: 119-140.

Joshi, A. C. 1946. A note on the development of pollen of Myristica fragrans and the affinities of the family Myristicaceae. J. Indian Bot. Soc. 25: 139-143.

Kumari, G. K. 1963. The primary vascular system of gymnosperms. Ph.D. thesis. University of Michigan, Ann Arbor.

Marsden, M. P. F., And I. W. Bailey. 1955. A fourth type of nodal anatomy in dicotyledons illustrated by Clerodendron trichotomum. J. Arnold Arbor. 36: 1-51.

Ozenda, P. 1949. Recherches sur les dicotyledones ápocarpiques. Publ. Lab. de L'Ecole Norm. Sup. Sér. Biol. II. Paris.

Pant, D. D., And B. Mehra. 1964. Nodal anatomy in retrospect. Phytomorphology 14: 384-387.

Parameswaran, N. 1961. Ruminate endosperm in the Canellaceae. Current Sci. 30: 344-345.

ShutTs, C. F. 1960. Wood anatomy of Hernandiaceae and Gyrocarpaceae. Trop. Woods 113: 85-123.

SinnotT, E. W. 1914. Investigations on the phylogeny of the angiosperms. I. The anatomy of the node as an aid in the classification of angiosperms. Amer. J. Bot. 1: 303-322.

Stern, W. L. 1954. Comparative anatomy and phylogeny of Lauraceae. Trop. Woods 100: 1-73.

Swamy, B. G. L. 1949. Further contributions to the morphology of the Degeneriaceac. J. Arnold Arbor. 30: $10-38$.

Tucker, S. C. 1962. Ontogeny and phyllotaxis of the terminal vegetative shoots of Michelia fuscata. Amer. J. Bot. 49: 722-737.

Vander Wyk, R. W., and J. E. Canright. 1956. The anatomy and relationships of the Annonaceae. Trop. Woods 104: 1-24.

Wrison, T. K. 1960. The comparative morphology of the Canellaceae. I. Synopsis of genera and wood anatomy. Trop. Woods 112: 1-27. 